\title{
A Study on Mouse Movement Features to Identify User
}

\author{
Masud Karim \\ Department of Computer Science and Engineering \\ University of Dhaka \\ Dhaka, Bangladesh \\ masud.mkarim@gmail.com \\ Md. Hasanuzzaman \\ Department of Computer Science and Engineering \\ University of Dhaka \\ Dhaka, Bangladesh \\ hzamancsdu@yahoo.com \\ DOI: $10.31364 / \mathrm{SCIRJ} / \mathrm{v} 8 . i 4.2020 . P 0420766$ \\ http://dx.doi.org/10.31364/SCIRJ/v8.i4.2020.P0420766
}

\begin{abstract}
Identifying real user is a vital issue in information security. Various processes are using to identify user like password, punch card, PIN etc which identify some characters of a user but not the real user. Biometric identification techniques can identify real user like face decoction, finger print, iris technology etc but these are cost effective and not user friendly due to requirements of extra devices and systems. For this reason, researchers are giving emphasis on mouse movement based user identification technique. This technique solves current problems of real user identification and need no extra device. In this technique features are generated from mouse movement data and based on these features users are identified. Different researchers are proposing different types of features for identifying user from mouse movement data. Selecting and calculating these features is very important issue for this technique. In this paper we have shown such features collectively those are using by different researchers. We have tried to categorized and describe such features in different types with accuracy. Recommendation is mentioned in this paper for further using of mouse movement features.
\end{abstract}

Keywords - user identification, authentication, classification, mouse movement features, machine learning.

\section{BACKGROUND STUDY}

User identification and authentication is important because it enables organizations to keep their networks secure by permitting only authenticated users to access its protected resources, which may include computer systems, networks, databases, websites and other network-based applications or services.

Most of the user identification or authentication systems are able to identify a user information but unable to identify original user. Mouse movement based user identification or authentication system ensures the presence of original user.
Because in this system, user access information is so critical that it cannot be stolen, hacked or known by force.

After the authentication user is authorized for several resources. Sometimes user is again authenticated for authorization. The conventional authentication systems are unable for continuous authentication. But mouse movement based system can handle the continuous authentication based on user mouse movement behavior.

The user identification using mouse movement data is an alternative that can identify user behavior accurately without any cost of additional device. There are several researchers working in the field of user authentication and identification using mouse movement data. Actually mouse dynamic based authentication is a new concept [3] [10]. Researchers are trying to formalize this concept.

Mouse movement data contains user unique behavior. Features of mouse movement data are important key to identify a user. Target of this research is to study and analysis features based on mouse movement data.

\section{RELATED RESEARCH}

S. Suganya and others [2] have addressed a method that creates a database of containing mouse dynamic data like co-ordinate value time stamp value and mouse operation. From these features, features vectors are obtained. The dataset contains the static mouse behavior data of 20 users. The dataset is collected from openly available data set. Diffusion map algorithm was proposed to reduce the dimension of future vectors. The author used neural network classes which made up of a number of simple and highly interconnected processing elements. Performance analysis is shown by comparing existing of proposed system as FAR and FRR where the proposed system shows $\mathrm{FAR}=4.15 \%$ and $\mathrm{FAR}=5.05 \%$. The proposed system shows better performance. 
Nazirah Abd Hamid etc. [6] captured data by using mouse clicks on several buttons. When user clicks a button then it vanishes and appears new one randomly. A user was asked to perform this experiment six times to establish the user personal profile consisting of user behaviors and characteristics. Raw data consisted of coordinate $\mathrm{X}$ and $\mathrm{Y}$ as the user clicked on a button that set in a certain grids. The other attribute that involved in the process was time in milliseconds. The raw data, $x$ and $y$ coordinates and time from a data file was applied with five different formulas to create a user's mouse profile. Then the average and standard deviation of each result would be determined as the mouse profile measurement.

Soumik Mondol and Patric Bours [27] have published a biometric authentication based on mouse dynamic and keystroke. By using software, they have collected mouse movement and keystroke data from 53 users. They have worked on continuous authentication system by developing robust trusted algorithm. Some new mouse movement features are introduced. A windows based login tools is created to collect data. Data are collected in csv format in local machine. They have converted mouse events into four different actions, single click, double click, move and drag-drop. Collected data are separated into three parts as, for training, to adjust parameter and for testing. Researchers have compared their result with related research and found their proposed system shows better result. They have also illustrated comparative result between continuous authentication and periodic authentication. Separate classification technics for different modalities along with different verification processes are applied. Researchers have used three different classifier models in Multi Classifier Fusion architecture for all the different actions. The regression models are Artificial Neural Network (ANN) and Counter Propagation Artificial Neural Network and the prediction model is Support Vector Machine (SVM).

Chinmayee.KS and Vanishree C [28] have proposed a framework to authenticate user from mouse movement data. The framework covers four modules: gesture creation, data acquisition and preprocessing, feature extraction and classification. They have worked for static authentication. Mouse movement data are analyzed using a Hidden Markov Model. They have captured mouse movement data by asking user to draw a mouse gesture. First gestures are stored as template. In next time user replaced gesture several times and these compared with template. The researchers have recommended to use their proposed system in complex environment like e commerce or e learning.

Anam Khan and others [29] have recently published a survey of performance analysis of mouse movement based user authentication research. They have compared the result based on FAR and FRR. Mouse dynamic features are shown during their study and some basic features are identified. Algorithms and classifications used in various related features are briefly describes. A snapshot is found from this paper for using tools and techniques of using mouse movement based authentication.

\section{ENVIRONMENT OF MOUSE MOVEMENT DATA}

Mouse movement features are generated from mouse movement data. Mouse movement data area collected based on mouse gesture, coordinate, start time, end time, time interval, idle time, drag-drop etc. Many researchers have worked with mouse movement data. Their focusing criteria/issues are also differing from researchers to researchers. It is very difficult to formalize all concepts together in single platform. Through, we can divide previous finding in two main categories:

i. $\quad$ Guided Mouse Movement

ii. Non Guided Mouse Movement

Guided Mouse Movement: This is a guided work, where all the users perform similar task with mouse. The users are asked to do a particular task and in background of this task user's mouse movement data are captured. Generally, user interface is developed for this environment to capture mouse movement data [2] [6] [19].

Non Guided Mouse Movement: It is a free style work where a user is being not guided for particular task. User can do any work with mouse and in his background mouse movement data are captured. In this style there is no guidance for user to move the mouse. There is no specific user interface for the user. In this style user is independent to move the mouse [7] [11] [25] [26].

\section{FEATURES OF MOUSE MOVEMENT DATA}

Different researchers have derived different features regarding mouse movement pattern. Calculations of these features also differ from researchers to researchers. Lists of such features are shown in Table I. These features are generated from mouse movement data. It is proved that features make differentiated among users. So features those are sharper, can identify users more accurately. Moreover, using the more features play important role to identify users. These features are key indicator to identify user mouse movement pattern.

Table I

LIST OF MOUSE MOVEMENT FEATURES

\begin{tabular}{|l|c|}
\hline \multicolumn{1}{|c|}{ Features } & Source \\
\hline Mouse operation frequency & \\
Mouse silence ratio & \\
Movement elapsed time & \\
Movement offset & \\
Average movement speed & \\
Distribution of cursor position & {$[2]$} \\
Average movement distance & \\
Horizontal velocity & \\
Vertical velocity & \\
Tangential velocity & \\
Tangential acceleration & \\
Tangential jerk & \\
Slope angle & \\
Curvature & \\
\hline Time & \\
Speed & {$[6]$} \\
Acceleration & \\
Deviation & {$[7]$} \\
Angle of deviation & {$[13]$} \\
\hline x-coordinate & {$[28]$} \\
y-coordinate & \\
time remaining features & \\
Horizontal velocity & \\
\hline
\end{tabular}

www.scirj.org

(C) 2020, Scientific Research Journal

http://dx.doi.org/10.31364/SCIRJ/v8.i4.2020.P0420766

This publication is licensed under Creative Commons Attribution CC BY. 


\begin{tabular}{|c|c|}
\hline Features & Source \\
\hline $\begin{array}{l}\text { Vertical velocity } \\
\text { Tangential velocity } \\
\text { Tangential acceleration } \\
\text { Tangential jerk } \\
\text { Path from the original pixel } \\
\text { Slope angle of the tangent } \\
\text { Curvature } \\
\text { Curvature rate of change }\end{array}$ & \\
\hline $\begin{array}{l}\text { Average between each pair of points } \\
\text { Standard deviation between each pair of points } \\
\text { Maximum between each point between each pair of points } \\
\text { Minimum between each point between each pair of points }\end{array}$ & [9] \\
\hline $\begin{array}{l}\text { Direction } \\
\text { Angle of curvature } \\
\text { Curvature distance }\end{array}$ & [11] \\
\hline $\begin{array}{l}\text { Movement speed over traveled distance per defined action } \\
\text { Movement speed per direction of movement } \\
\text { Acceleration per direction of movement }\end{array}$ & [15] \\
\hline $\begin{array}{l}\text { Down-Up time } \\
\text { Down-Down time } \\
\text { Up-Down time } \\
\text { Up-Up time }\end{array}$ & [16] \\
\hline $\begin{array}{l}\text { Size of a curve } \\
\text { Length of a Mouse curve } \\
\text { Total time of the Mouse curve } \\
\text { Mouse speed over a pre-defined action } \\
\text { Angle of Mouse Movement } \\
\text { Acceleration } \\
\text { Mouse click duration }\end{array}$ & $\begin{array}{l}{[17]} \\
{[17]}\end{array}$ \\
\hline $\begin{array}{l}\text { Speed over } 8 \text { directions } \\
\text { Speed over different distance(short, long and very long) }\end{array}$ & [19] \\
\hline $\begin{array}{l}\text { Mouse pressure } \\
\text { Electro galvanic skin conductance, } \\
\text { Skin temperature } \\
\text { Speed of mouse pointer's movement } \\
\text { Acceleration speed of mouse pointer's movement } \\
\text { Amplitude of hand trembles } \\
\text { Scroll wheel use } \\
\text { Right- and left-click frequency } \\
\text { Idle time }\end{array}$ & [21] \\
\hline $\begin{array}{l}\text { Number of events } \\
\text { For each event category and for the cursor movement } \\
\text { data, the mean, standard deviation and third moment of } \\
\text { the distance, angle and speed between pairs of points. } \\
\text { For each event category and for the cursor movement } \\
\text { data, we compute the mean, standard deviation and third } \\
\text { moment for the X and for the Y coordinates. }\end{array}$ & [22] \\
\hline $\begin{array}{l}\mathrm{X} \text { and y position value } \\
\text { Distance metrics(Euclidean distance, Manhattan distance } \\
\& \text { edit distance) }\end{array}$ & [23] \\
\hline $\begin{array}{l}\text { Number of trajectory points } \\
\text { Amount of time to complete trajectory } \\
\text { Length of trajectory } \\
\text { Velocity from point to point in the trajectory } \\
\text { Acceleration from point to point in the trajectory } \\
\text { Direction angle from point to point in the trajectory } \\
\text { Number of inflection points in the trajectory } \\
\text { Curviness of the trajectory }\end{array}$ & [24] \\
\hline $\begin{array}{l}\text { Time elapsed, } \\
\text { Distance traveled, } \\
\text { Direction or range or angle of each movement }\end{array}$ & [25] \\
\hline $\begin{array}{l}\text { Movement speed compared to traveled distance } \\
\text { Average movement speed per movement direction } \\
\text { Movement direction histogram } \\
\text { Average movement speed per types of actions } \\
\text { Action type histogram } \\
\text { Traveled Distance Histogram }\end{array}$ & [26] \\
\hline
\end{tabular}

\begin{tabular}{|l|c|}
\hline \multicolumn{1}{|c|}{ Features } & Source \\
\hline Movement elapsed time histogram & \\
\hline $\begin{array}{l}\text { Mouse single click action } \\
\text { Mouse double click action }\end{array}$ & \\
Mouse move action & [27] \\
Mouse drag-drop action & \\
\hline
\end{tabular}

We find many difference among features listed above those have been used by different researchers. In some cases, researchers have used same feature name but calculation to find the feature is not the same. On the other side similar calculation is used for different named features.

Sometimes features are captured based on specific environment. But in cloud computing or in a large network user working environment is unknown. Some features work only mouse movement angle [6]. But it is difficult to get frequently angle value from user mouse movement. There are some features those depends on specific mouse click event. But in a long session required clicks may not have occurred. There are also some mouse movement features those depend on keyboard typing pattern [4] [5]. In these cases, users are identified based on combination of user's work using mouse and keyboard.

\section{A. General and Extended Features}

After analysis the features used in current research we observed that we can generate many features from two general features. These are, time and pointer position.

1. Time: Time value can be taken from mouse movement as whenever clicks, idle, starting and ending of particular task, task for time interval etc.

2. Pointer Position: This is the $\mathrm{X}-\mathrm{Y}$ coordinate point of mouse pointer in certain time or certain click.

If we can find out the value of the above two general features, then other features can be easily calculated. The table II shows extended features and their relations with general features.

Table II

EXTENDED FEATURES FROM TIME FEATURE

\begin{tabular}{|c|c|}
\hline $\begin{array}{c}\text { General } \\
\text { Features -> }\end{array}$ & Time \\
\hline \multirow{14}{*}{ 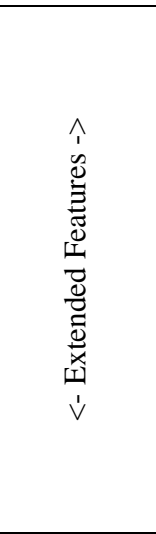 } & Movement elapsed time \\
\hline & Movement offset \\
\hline & time remaining features \\
\hline & Acceleration per direction of movement \\
\hline & Down-Up time \\
\hline & Down-Down time \\
\hline & Up-Down time \\
\hline & Up-Up time \\
\hline & Mouse click duration \\
\hline & Mouse pressure \\
\hline & Scroll wheel use \\
\hline & Idle time \\
\hline & Time elapsed \\
\hline & Movement elapsed time histogram \\
\hline
\end{tabular}

Table III

EXTENDED FEATURES FROM POINTER POSITION FEATURE

\begin{tabular}{|c|c|}
\hline $\begin{array}{c}\text { General } \\
\text { Features -> }\end{array}$ & Pointer Position \\
\hline \multirow{4}{*}{ 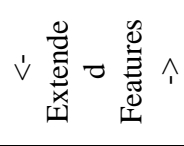 } & Mouse operation frequency \\
\hline & Distribution of cursor position \\
\hline & Average movement distance \\
\hline & Tangential jerk \\
\hline
\end{tabular}




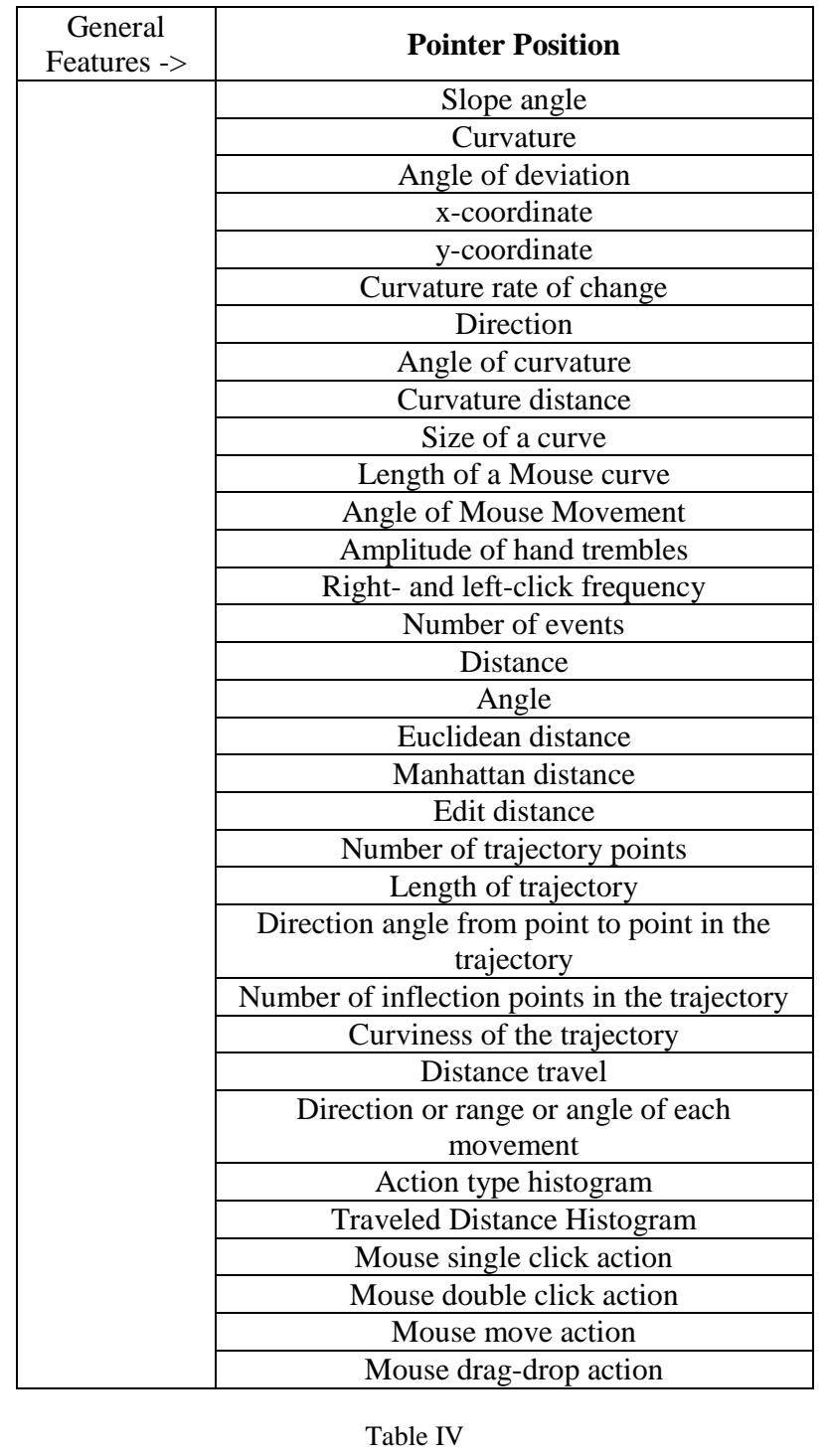

EXTENDED FEATURES FROM TIME \& POINTER POSITION FEATURES

\begin{tabular}{|c|c|}
\hline \multicolumn{2}{|r|}{ FEATUKES } \\
\hline $\begin{array}{c}\text { General } \\
\text { Features -> }\end{array}$ & Time \& Pointer Position \\
\hline \multirow{19}{*}{ 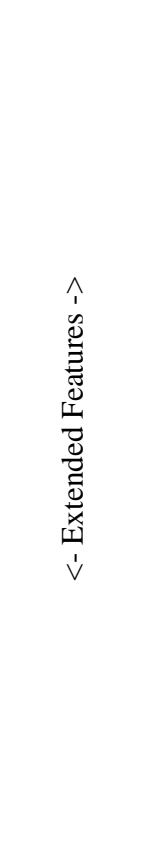 } & Mouse silence ratio \\
\hline & Average movement speed \\
\hline & Horizontal velocity \\
\hline & Vertical velocity \\
\hline & Tangential velocity \\
\hline & Tangential acceleration \\
\hline & Speed \\
\hline & Acceleration \\
\hline & Deviation \\
\hline & $\begin{array}{c}\text { Movement speed over traveled distance per } \\
\text { defined action }\end{array}$ \\
\hline & Movement speed per direction of movement \\
\hline & Total time of the Mouse curve \\
\hline & Mouse speed over a pre-defined action \\
\hline & Speed over 8 directions \\
\hline & $\begin{array}{l}\text { Speed over different distance(short, long and } \\
\text { very long) }\end{array}$ \\
\hline & Amount of time to complete trajectory \\
\hline & Velocity from point to point in the trajectory \\
\hline & $\begin{array}{l}\text { Acceleration from point to point in the } \\
\text { trajectory }\end{array}$ \\
\hline & $\begin{array}{l}\text { Movement speed compared to traveled } \\
\text { distance }\end{array}$ \\
\hline
\end{tabular}

\begin{tabular}{|c|c|}
\hline $\begin{array}{c}\text { General } \\
\text { Features }->\end{array}$ & Time \& Pointer Position \\
\hline \multirow{3}{*}{} & $\begin{array}{c}\text { Average movement speed per movement } \\
\text { direction }\end{array}$ \\
\cline { 2 - 2 } & $\begin{array}{c}\text { Average movement speed per types of } \\
\text { actions }\end{array}$ \\
\hline
\end{tabular}

It is mentioned that more extended features can be find out and calculated from the mentioned two general features.

\section{B. Comparison of Accuracy}

Since the user identification based on mouse movement data is concept of biometric authentication so most of researchers have used False Acceptance Rate (FAR) and False Rejection Rate (FRR) to check the accuracy of their system. A simple presentation of the accuracy of different systems are illustrated in figure 1. After studying many systems, we have seen that system sharpness is not only depends on FAR and FRR but also the system environment and amount of data are used for testing.

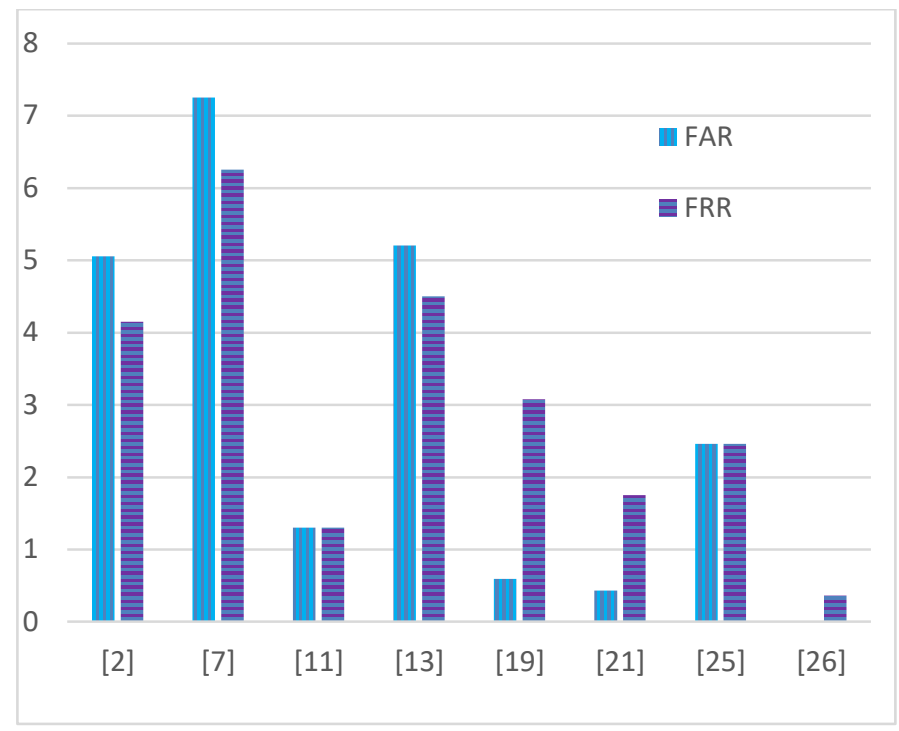

Fig 1: Preview of Accuracy level

\section{RECOMMENDATION FOR MOUSE MOVEMENT FEATURES}

After the analysis the mouse movement features and them strengthens, we are recommending to segregate the features. The more features can be find out the system should be more strong to identified user. After finding a feature we can segregate it into more features. On the other hand, non-guided environment as discussed in section III is more effective to detect user whenever more user in large system and continuous user identification is needed. Guided environment is more effective whenever less user in a small system. From Figure 1 we have found that researches [11], [35] and [40] show better performance and for these references data are captured from non-guided system.

\section{CONCLUSION}

Cloud computing is increasing rapidly day by day. As a result, security is getting top priority to use systems and access resources. So, strong authentication and identification is essential to determine presence of user. In this research we have tried to show mouse movement features in single 
framework that can be used for further research for related analysis and further development.

\section{ACKNOWLEDGMENT}

This project is done at Department of Computer Science and Engineering, University of Dhaka, Bangladesh. We thank to ICT Division, Ministry of Posts, Telecommunications and IT, Government of the People's Republic of Bangladesh for funding.

\section{REFERENCE}

[1] Ross A.J.Everittand Peter W.McOwan; "Java-Based Internet Biometric Authentication System"; IEEE Transactions on Pattern Analysis And Machine Intelligence, Vol.25, No.9, September2003.

[2] S. Suganya, G. Muthumari, and C. Balasubramanian; "Improving the Performance of Mouse Dynamics Based Authentication Using Machine Learning Algorithm"; International Journal of Innovation and Scientific Research; ISSN 2351-8014 Vol. 24 No. 1 Jun. 2016, pp. 202-209.

[3] Mr. Vishal S. Patil; Prof. P.R.Devale; "Biometric Validation by Storing different Patterns using Mouse Gesture Signatures"; International Journal on Recent and Innovation Trends in Computing and Communication; Volume: 3 Issue: 4; April 2015.

[4] AnandMotwani, Raina Jain, JyotiSondhi; "A Multimodal Behavioral Biometric Technique for User Identification using Mouse and Keystroke Dynamics"; International Journal of Computer Applications (0975 - 8887); Volume 111 - No 8, February 2015.

[5] Raina K. Jain, SanchetiShital D, PansareJyoti T, KawaleShubhangi K; "Mouse and Keystroke Based Behavioural Biometrics: Techniques, Problems and Solutions"; International Journal of Modern Trends in Engineering and Research; e-ISSN No.:2349-9745, Date: 2-4 July, 2015

[6] NazirahAbd Hamid, SuhailanSafei, SitiDhalilaMohdSatar, SuriayatiChuprat and Rabiah Ahmad; "Randomized Mouse Movement for Behavioral Biometric Identification," International Journal of Interactive Digital Media, Vol. 1(2), ISSN 2289-4098, eISSN 2289-4101, 2013.

[7] G. Muthumari, R. Shenbagaraj, M. BlessaBinolin Pepsi; "Mouse Gesture Based Authentication Using Machine Learning Algorithm," 2014 IEEE International Conference on Advanced Communication Control and Computing Technologies (ICACCCT)

[8] A. Kaklauskas, E. K. Zavadskas, M. Seniut, M. Krutinis, G. Dzemyda, S. Ivanikovas, V. Stankevič, Č. Šimkevičius, A. Jaruševičius; "Web-Based Biometric Mouse Decision Support System For User's Emotional And Labour Productivity Analysis"; The 25th International Symposium on Automation and Robotics in Construction; June 26-29, 2008; PP-69-75

[9] Shivani Hashia, "Authentication by Mouse Movements", San Jose State University.

[10] Dr. Jien (Morris) Chang, Mr. Kuan-Hsing Ho and Mr. Chi-Chen Fang, Capturing Cognitive Fingerprints from Keystroke Dynamics for Active User Authentication, all of the Department of Electrical and Computer
Engineering, Iowa State University of Science and Technology.

[11] Nan Zheng, Aaron Paloski, and Haining Wang; An Efficient User Verification System via Mouse Movements; Department of Computer Science, The College of William and Mary Williamsburg, VA 23185, USA;

[12] Joseph S. Valacich, Jeffrey L. Jenkins, Jay F. Nunamaker, Jr, Salim Hariri, John Howie; Identifying Insider Threats through Monitoring Mouse Movements in Concealed Information Tests; University of Arizona.

[13] Chethan D C, Mr.Sundaresh M P; Design and Development of a Biometric System Using Mouse Gesture Dynamics; International Journal of Advanced Research in Computer Engineering \& Technology (IJARCET).

[14] Mounashree H $\quad$ C, Nancy $\quad$ M R,NavyaM,NirupamaBLinganagoudar,Mangala $\mathrm{C} \quad \mathrm{N}$; MouseGesture for Authentication by Hidden Markov Model; International Journal of Computer, Information Technology \& Bioinformatics (IJCITB), ISSN: 22787593, Volume-2, Issue-2.

[15] Chee-Hyung Yoon, Daniel Donghyun Kim; User Authentication Based On Behavioral Mouse Dynamics Biometrics; Department of Computer Science, Stanford, Stanford, CA 94305.

[16] Cheng-Jung Tsai, Ting-Yi Chang, Yu-Ju Yang, MengSung $\mathrm{Wu}$, Yu-Chiang Li; An Approach For User Authentication On Non-Keyboard Devices Using Mouse Click Characteristics and Statistical-Based Classification; International Journal of InnovativeComputing, Information and Control, ISSN 1349-4198, Volume 8, Number 11, November 2012 pp. 7875-7886.

[17] Adam Weiss, Anil Ramapanicker, Pranav Shah, Shinese Noble and Larry Immohr; Mouse Movements Biometric Identification: A Feasibility Study; Faculty Research Day, CSIS, Pace University, May 4th, 2007.

[18] NkemAjufor, Antony Amalraj, Rafael Diaz, Mohammed Islam, Michael Lampe; Refinement of a Mouse Movement Biometric System; Ivan G Seidenberg School of CSIS, Pace University, 1 Martine Ave, White Plains, NY, 10606, USA.

[19] Saurabh Singh, Dr. K.V.Arya; Mouse Interaction based Authentication System by Classifying the Distance Travelled by the Mouse; International Journal of Computer Applications (0975 - 8887), pp-45-48, Volume 17- No.1, March 2011.

[20] Zach Jorgensen and Ting Yu; On Mouse Dynamics as a Behavioral Biometric for Authentication; ASIACCS '11, March 22-24, 2011, Hong Kong, China. Pp476-482.

[21] MajaPusara, Carla E. Brodley; User Re-Authentication via Mouse Movements; VizSEC/DMSEC'04, October29,2004, Washington, DC, USA.Copyright 2004 ACM 1-58113-974-8/04/0010.

[22] Govardhini.S, Kowshika.A; An Effective User Recognition Using Mouse Gesticulation; International Journal of Advanced Research inComputer Science and Software Engineering; Volume 3, Issue 11, November 2013; ISSN: 2277 128X; pp 456 - 460.

[23] HediehZandikarimi, Frank Lin, Celia Carlos, Justin Correa, Phil Dressner, and Vinnie Monaco; Design of a Mouse Movement Biometric System to Verify the 
Identity of Students Taking Multiple-Choice Online Tests; Seidenberg School of CSIS, Pace University, White Plains, New York

[24] Mr. Vishal S. Patil, Prof. P.R.Devale; "Biometric Validation by Storing different Patterns using Mouse Gesture Signatures"; International Journal on Recent and Innovation Trends in Computing and Communication; Volume: 3 Issue: 4, April 2015

[25] Ahmed Awad E.Ahmed and Issa Traore, Member, IEEE; A New Biometric Technology Basedon Mouse Dynamics; IEEE Transactions on dependable and secure computing, Vol.4, No.3, July-September 2007.

[26] Youssef Nakkabi, IssaTraoré and Ahmed AwadE.Ahmed; Improving Mouse Dynamics Biometric Performance Using Variance Reduction via Extractors with Separate Features; IEEE Transactions On Systems, Man, And Cybernetics — Part A: Systems And Humans, Vol.40, No.6, November 2010

[27] Soumik Mondal, Patrick Bours; "A study on Continuous Authentication using a combination of Keystroke and Mouse Biometrics"; ELSEVIE journal, Article in Neurocomputing, November 2016; DOI: 10.1016/j.neucom.2016.11.031

[28] Chinmayee.KS, Vanishree C; "Continuous Authentication for Mouse Gesture Recognition using Hidden Markov Model"; InternatIonal Journal of electronIcs \& communIcatIon technology; Vol- 8, Issue 4 OCT - DEC 2017.

[29] Anam Khan, Suhail Javed Quraishi, Sarabjeet Singh Bedi; "Mouse Dynamics as Continuous User Authentication Tool"; International Journal of Recent Technology and Engineering (IJRTE), ISSN: 2277-3878, Volume-8 Issue-4, November 2019 